\title{
Lesão do manguito rotador e obesidade: Uma avaliação demográfica e metabólica*
}

\section{Rotator Cuff Lesion and Obesity: A Demographic and Metabolic Evaluation}

\author{
Saulo Teixeira Pansiere ${ }^{1,2(0)}$ Arlane Carvalho de Oliveira ${ }^{1}$ Alberto de Castro Pochini ${ }^{2}$ A \\ Benno Ejnisman ${ }^{2 \odot}$ Paulo Santoro Belangero ${ }^{2 \odot}$ Carlos Vicente Andreoli ${ }^{2 \odot}$ \\ ${ }^{1}$ Instituto de Pesquisa e Ensino do Hospital Ortopédico e Medicina \\ Especializada (IPE-HOME), Brasília, DF, Brasil \\ ${ }^{2}$ Centro de Traumato-ortopedia do Esporte (Cete), Universidade \\ Federal de São Paulo (Unifesp), São Paulo, Brasil \\ Endereço para correspondência Saulo Teixeira Pansiere, MD, Hospital \\ Ortopédico e Medicina Especializada (HOME), SGAS Quadra 613, \\ Conjunto C, Brasília, DF, 70200-730, Brasil \\ (e-mail: saulopansiere@hotmail.com).
}

Rev Bras Ortop 2022;57(2):282-288.

\begin{abstract}
Resumo
Palavras-chave

- manguito rotador

- obesidade

- índice de massa corporal

- diabetes

- hipertensão arterial

- ultrassonografia

Objetivo Analisar a relação da presença e da gravidade da lesão do manguito rotador (MR) com a obesidade e o tempo de exposição à obesidade. De forma secundária, avaliar a relação e a prevalência de fatores demográficos e metabólicos em indivíduos obesos com lesão do MR.

Métodos Trata-se de um estudo transversal, com 235 pacientes obesos (índice de massa corporal $[\mathrm{IMC}] \geq 30 \mathrm{~kg} / \mathrm{m}^{2}$ ). Dados demográficos (idade e gênero), metabólicos (hipertensão, diabetes mellitus, perfil lipídico, e tempo de exposição à obesidade), exame físico (peso, estatura, circunferência abdominal, e testes clínicos), e exame ultrassonográfico musculoesquelético foram utilizados para a análise dos resultados. Resultados Não foi evidenciada associação da lesão do MR com IMC $(p=0,82)$, tempo de exposição à obesidade $(p=0,29)$, ou circunferência abdominal $(p=0,52)$. No subgrupo com lesão, a idade $(p<0,001)$, a presença de diabetes melito $(p=0,013)$, a hipertensão $(p<0,001)$, o nível de lipoproteína de alta densidade (high-density lipoprotein, $\mathrm{HDL}$, em inglês) $(p=0,026)$, e o tempo de exposição à obesidade $(p<0,001)$ foram significativamente maiores em comparação ao subgrupo sem lesão do MR. Na busca por demais parâmetros associados de forma independente para lesão do MR, foram observadas associações com idade $(p=0,0003)$ e hipertensão ( $p$ $=0,004)$.
\end{abstract}

Trabalho desenvolvido no Centro de Traumato-ortopedia do Esporte (Cete), Universidade Federal de São Paulo (Unifesp), São Paulo, Brasil.

recebido

09 de Maio de 2020

aceito

01 de Dezembro de 2020

Publicado on-line

de Janeiro 27, 2022
DOI https://doi.org/

10.1055/s-0041-1729937.

ISSN $0102-3616$. (c) 2022. Sociedade Brasileira de Ortopedia e Traumatologia. All rights reserved.

This is an open access article published by Thieme under the terms of the Creative Commons Attribution-NonDerivative-NonCommercial-License, permitting copying and reproduction so long as the original work is given appropriate credit. Contents may not be used for commercial purposes, or adapted, remixed, transformed or built upon. (https://creativecommons.org/ licenses/by-nc-nd/4.0/)

Thieme Revinter Publicações Ltda., Rua do Matoso 170, Rio de Janeiro, RJ, CEP 20270-135, Brazil 
Abstract

\section{Keywords}

- rotator cuff

- obesity

- body mass index

- diabetes

- arterial hypertension

- ultrasonography
Conclusão Não evidenciamos associação da obesidade e do tempo de exposição a ela com a ocorrência e a gravidade da lesão do MR. Porém, indivíduos com lesão apresentaram maior tempo de exposição à obesidade e prevalência de disfunções metabólicas do que indivíduos sem lesão. Além disso, nossos achados sugerem uma associação entre hipertensão arterial sistêmica (HAS) e idade avançada com a lesão do MR.

Objective To analyze the relationship between the presence and severity of rotator cuff (RC) injury with obesity and the time of exposure to obesity. Secondarily, to evaluate the relationship and prevalence of demographic and metabolic factors in obese individuals with RC injury.

Methods This is a cross-sectional study with 235 obese patients (body mass index $[\mathrm{BMI}] \geq 30 \mathrm{~kg} / \mathrm{m}^{2}$ ). Demographic data (age and gender), metabolic data (hypertension, diabetes mellitus, lipid profile, and time of exposure to obesity), physical examination (weight, height, waist circumference, and clinical tests), and musculoskeletal ultrasound examination were used to analyze the results.

Results There was no evidence of an association between RC injury and BMI $(p=0.82)$, time of exposure to obesity $(p=0.29)$, or abdominal circumference $(p=0.52)$. In the subgroup with injury, age $(p<0.001)$, presence of diabetes mellitus $(p=0.013)$, hypertension $(p<0.001)$, level of high-density lipoprotein (HDL) $(p=0.026)$, and time of exposure to obesity $(p<0.001)$ were significantly greater compared to the subgroup without injury. In the search for other parameters independently associated with RC injury, associations were observed with age $(p=0.0003)$ and hypertension $(p=0.004)$.

Conclusion We did not evidence an association between obesity and the time of exposure to it with the occurrence and severity of RC injury. However, individuals with injury had a longer time of exposure to obesity and prevalence of metabolic disorders than individuals without RC injury. In addition, our findings suggest an association between systemic arterial hypertension (SAH) and advanced age with RC injury.

\section{Introdução}

A obesidade, definida pela Organização Mundial de Saúde (OMS) como o acúmulo anormal ou excessivo de gordura corporal, é considerada um problema de saúde pública devido à alta prevalência e às consequências associadas a ela. ${ }^{1-3} \mathrm{~A}$ incidência dessa condição aumentou exponencialmente nos últimos anos, e atinge mais de $10 \%$ da população mundial. ${ }^{4}$ No Brasil, entre 2006 e 2018, houve um aumento do número de indivíduos obesos superior a $67 \%{ }^{5}$

Pesquisas vêm demonstrando que a elevação no índice de massa corporal (IMC) é um importante fator relacionado a doenças metabólicas crônicas (diabetes melito tipo 2 [DM2], hipertensão arterial sistêmica [HAS] e dislipidemia), ${ }^{6-8}$ e a certos transtornos do sistema musculoesquelético. ${ }^{9-11} \mathrm{O}$ excesso de peso corporal está associado a um aumento do risco de desenvolver doenças cardiovasculares e o início precoce da morbidade cardíaca. ${ }^{1,6-8}$ Variáveis de análise da obesidade, como o IMC e a circunferência abdominal, são consideradas fatores independentes e modificáveis associados a HAS, DM2 e dislipidemia. ${ }^{1,2,12}$

Além de tais correlações já difundidas cientificamente, a obesidade parece contribuir para lesões tendíneas, como as do manguito rotador (MR), predispondo o tendão a degene- rações e rupturas. ${ }^{9-11,13}$ A plausibilidade biológica para tal associação pode estar ligada à liberação de adipocinas próinflamatórias, o que leva a um estresse oxidativo e um estado de inflamação sistêmica crônica. ${ }^{9}{ }^{14}$ Considera-se que outros fatores metabólicos (DM e HAS) e demográficos (idade e gênero) podem estar associados à causalidade ou ao agravamento de tendinopatias, e, portanto, são foco de estudos recentes. ${ }^{15,16} \mathrm{O}$ tendão de indivíduos diabéticos é caracterizado por aumento da espessura e do volume, com desorganização das fibras de colágeno. ${ }^{17,18}$ Tais anormalidades parecem ser resultado da diminuição do fluxo sanguíneo periférico e da angiogênese local. ${ }^{15}$ Além disso, os danos ligados à HAS, como a lesão de microvasos, podem agravar as deficiências vasculares da zona crítica do MR, levando à hipóxia e à produção de espécies reativas de oxigênio, culminando em apoptose celular e degeneração tecidual. ${ }^{19,20}$

Apesar de tais constatações, a literatura científica ainda é escassa em estudos que relacionem valores altos de IMC (> $30 \mathrm{~kg} / \mathrm{m}^{2}$ ), circunferência abdominal, e tempo de exposição à obesidade às lesões dos tendões do MR em populações brasileiras. Ademais, a controvérsia científica persiste, uma vez que outros estudos ${ }^{21,22}$ não relataram tal associação (entre obesidade e lesões do MR). Da mesma forma, as relações das lesões 
do MR em indivíduos obesos com fatores metabólicos e demográficos ainda não foram completamente estabelecidas.

Diante disso, hipotetizamos que a ocorrência e a gravidade da lesão do MR estão associadas à obesidade (avaliada pelo IMC e pela circunferência abdominal) e ao tempo de exposição à obesidade. De forma secundária, acreditamos que fatores demográficos e metabólicos estão relacionados com a lesão do MR, além de existir maior prevalência de disfunções metabólicas nos indivíduos com lesão em comparação com aqueles sem lesão. Para testar essas hipóteses, realizamos um estudo transversal em pacientes adultos obesos.

\section{Metodologia}

\section{Desenho do estudo e participantes}

A população-alvo deste estudo transversal foi composta por homens e mulheres pacientes do ambulatório de endocrinologia e obesidade de nossa instituição entre 2018 e 2019.

Foram incluídos pacientes que apresentaram IMC $>30$ $\mathrm{kg} / \mathrm{m}^{2}$ e idade entre 18 e 65 anos. Foram excluídos do estudo voluntários que apresentaram fatores de risco diretos para lesão do $\mathrm{MR}$, como trauma prévio, tabagismo, cirurgia de ombro, instabilidade glenoumeral, uso crônico de corticoides, infiltrações, artrite reumatoide, artrose glenoumeral avançada, tendinopatia calcária, qualquer outro diagnóstico de calcificação, e que não concordaram em assinar o termo de consentimento livre e esclarecido (TCLE) ou não completaram todas as etapas do estudo.

O tamanho da amostra ( $\mathrm{n}$ ) necessário para este estudo foi calculado usando-se o intervalo de confiança de 95\% (IC95\%) e significância de $5 \%$, considerando a maior variância (25\%). Foi relevante o o número de pacientes (mil) atendidos na instituição no período de 12 meses, que consistiu a população-alvo. $O$ cálculo do tamanho da amostra foi efetuado com base na estimativa de proporções. ${ }^{23}$ Diante disso, para que houvesse relevância, deveriam ser pesquisados 235 indivíduos.

A amostra original da pesquisa foi constituída por 329 indivíduos. Desses, $94(28,6 \%)$ foram excluídos por não completarem todas as etapas do estudo, e restaram 235 $(71,4 \%)$ indivíduos qualificados (-Figura $\mathbf{1}$ ).

\section{Aprovações éticas}

Esta pesquisa foi aprovada pelo Comitê de Ética em Pesquisa sob o número de parecer 3.733.973. Todos os participantes eram voluntários e assinaram o TCLE, dando ciência de que conheciam os procedimentos a serem realizados e concordavam com os objetivos da pesquisa.

\section{Viés}

Cuidado foi tomado para evitar mal-entendidos nas respostas dos participantes. Os investigadores se abstiveram de apresentar quaisquer comentários que pudessem induzir a respostas contrárias à verdade passada pelos pacientes.

\section{Dados coletados e avaliações}

Os participantes do estudo foram entrevistados inicialmente para coleta de fatores demográficos (idade e gênero) e

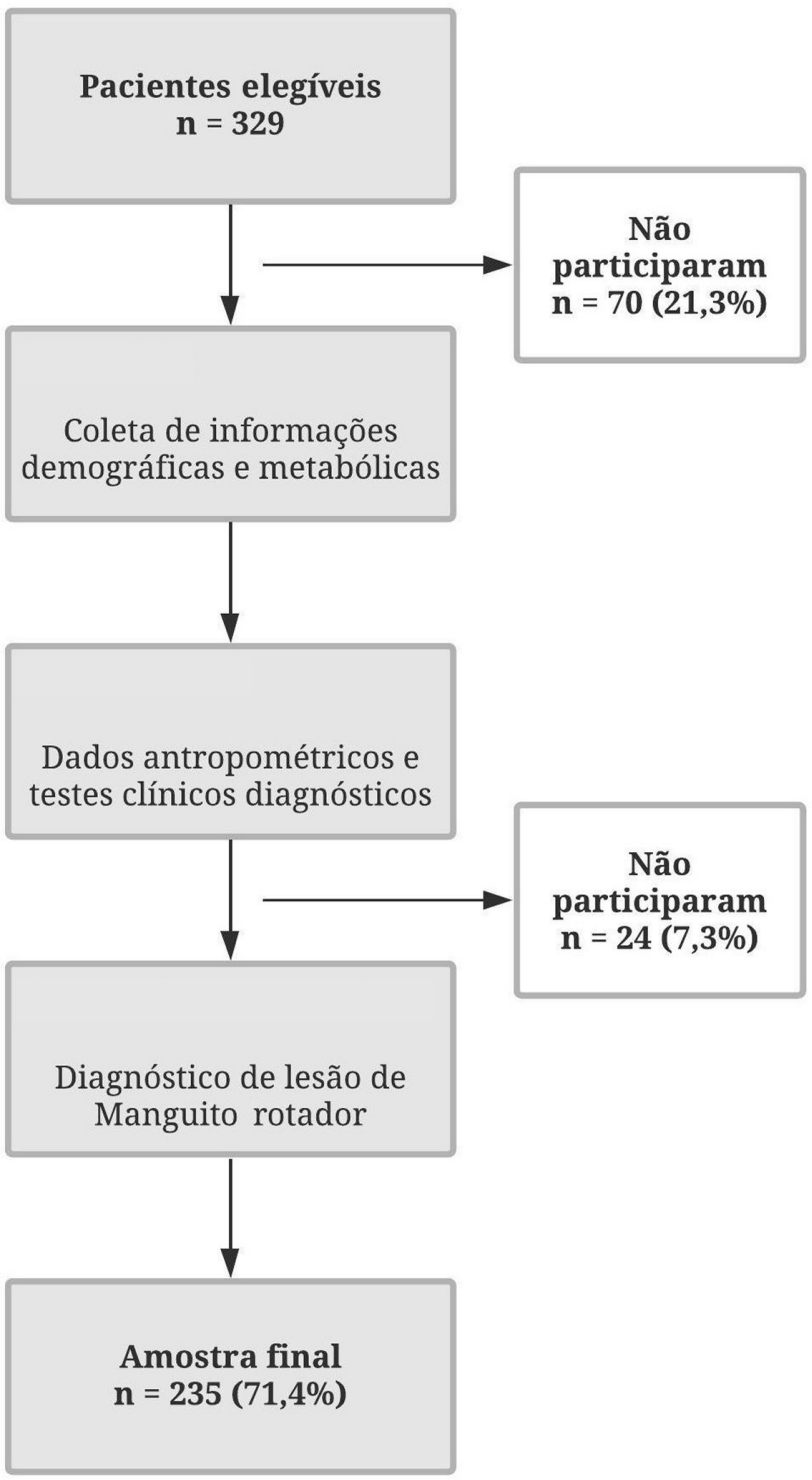

Fig. 1 Diagrama de fluxo dos pacientes do estudo.

metabólicos (prevalência de HAS, DM, perfil lipídico, e tempo de exposição à obesidade).

O exame físico envolveu a avaliação dos dados antropométricos de peso e estatura, realizados com os pacientes sem sapatos e vestindo roupas leves (à partir desses dados, o IMC individual foi calculado), circunferência abdominal, e testes clínicos norteadores de diagnóstico da lesão do MR (testes de Jobe, de Patte, e de Gerber), realizados conforme suas descrições. ${ }^{24-26}$ Para fechar o diagnóstico de lesão completa ou parcial do MR, o exame ultrassonográfico musculoesquelético foi realizado bilateralmente, em todos os pacientes, utilizando o protocolo estabelecido por Selvaraj et al. ${ }^{27} \mathrm{~A}$ ultrassonografia é um método confiável, que apresenta elevada acurácia no diagnóstico das lesões do MR. ${ }^{28}$

Os exames físicos dos voluntários foram realizados por médico com residência em ortopedia e traumatologia, especialista em cirurgia de ombro/cotovelo, no ambulatório de endocrinologia e obesidade. Os exames de imagem foram 
Tabela 1 Características demográficas, metabólicas e físicas da amostra em estudo

\begin{tabular}{|c|c|c|c|}
\hline & $\mathbf{n}$ & Média & ( \pm Desvio padrão) \\
\hline \multicolumn{4}{|l|}{ Fatores demográficos } \\
\hline Gênero feminino & 157 & & \\
\hline Gênero masculino & 78 & & \\
\hline Idade (anos) & & 40,5 & 11,1 \\
\hline \multicolumn{4}{|l|}{ Fatores metabólicos } \\
\hline LDL (mg/dL) & & 101 & 35 \\
\hline $\mathrm{HDL}(\mathrm{mg} / \mathrm{dL})$ & & 49,4 & 11,9 \\
\hline Colesterol total $(\mathrm{mg} / \mathrm{dL})$ & & 183 & 38 \\
\hline Razão de hipertensão arterial sistêmica (sim/não) & $85 / 150$ & & \\
\hline Razão de diabetes melito (sim/não) & $42 / 193$ & & \\
\hline $\begin{array}{l}\text { Tempo de exposição } \\
\text { à obesidade (anos) }\end{array}$ & & 11,7 & 8,1 \\
\hline \multicolumn{4}{|l|}{ Fatores físicos } \\
\hline Peso $(\mathrm{kg})$ & & 102 & 22 \\
\hline Altura $(\mathrm{cm})$ & & 166 & 10 \\
\hline Índice de massa corporal $\left(\mathrm{kg} / \mathrm{m}^{2}\right)$ & & 36,8 & 5,7 \\
\hline Circunferência abdominal $(\mathrm{cm})$ & & 114 & 15 \\
\hline
\end{tabular}

Abreviaturas: LDL, low-density lipoprotein (lipoproteína de baixa densidade); HDL, high-density lipoprotein (lipoproteína de alta densidade).

desempenhados por médico especialista em Ultrassonografia Musculoesquelética, com título expedido pela Associação Médica Brasileira na área de radiologia e diagnóstico por imagem. Ambos eram avaliadores independentes, sem vínculos empregatícios com as instituições envolvidas, e desconheciam qualquer informação relacionada aos objetivos da pesquisa.

\section{Definições}

O IMC foi calculado com base no peso em quilogramas dividido pela altura em metros quadrados. A definição de obesidade se deu com um IMC $>30 \mathrm{~kg} / \mathrm{m}^{2}$. A análise da circunferência abdominal foi realizada com a mensuração de 0,5 c, a $1,0 \mathrm{~cm}$ acima do umbigo com os sujeitos respirando naturalmente. $\mathrm{O}$ tempo de exposição à obesidade foi calculado levando-se em consideração a primeira vez que o participante atingiu o IMC $>30 \mathrm{~kg} / \mathrm{m}^{2}$ (avaliado por meio de análise da história pregressa, relatada pelo próprio paciente).

O DM foi definido como um nível glicose plasmática em jejum de 7,0 mmol/L, ou por um diagnóstico prévio realizado por profissional médico. A HAS foi definida de acordo com os seguintes critérios: pressão arterial sistólica de $140 \mathrm{mmHg}$, e pressão arterial diastólica de $90 \mathrm{mmHg}$ e/ou hipertensão autorreferida. O perfil lipídico foi analisado com base no colesterol total, na lipoproteína de baixa densidade (lowdensity lipoprotein, LDL, em inglês), e na lipoproteína de alta densidade (high-density lipoprotein, HDL, em inglês) por lipidograma completo feito até seis meses antes do estudo.

\section{Estatística}

As características demográficas, metabólicas e físicas da amostra foram calculadas usando estatística descritiva, incluindo frequências, médias e desvios padrão (DSs). A fim de comparar as variáveis demográficas, metabólicas e físicas dos pacientes com e sem lesão do MR, dois grupos foram criados (com lesão do $M R$ e sem lesão do MR) e analisados por meio do teste $t$ de Student e pelo teste do qui quadrado. Uma análise multivariada que identificou as variáveis demográficas, metabólicas e físicas associadas à lesão do MR foi realizada pela regressão logística binária com

Tabela 2 Perfil das lesões do manguito rotador da amostra em estudo

\begin{tabular}{|l|l|l|}
\hline \multicolumn{2}{|l|}{ n } & $\%$ \\
\hline Presença de lesão & 55 & $23,4 \%$ \\
\hline Sim & 180 & $76,6 \%$ \\
\hline Não & \multicolumn{2}{|l|}{} \\
\hline Membro da lesão & 32 & 58,2 \\
\hline Direito & 7 & 12,7 \\
\hline Esquerdo & 16 & 29,1 \\
\hline Ambos & \multicolumn{2}{|l|}{} \\
\hline Tipo de lesão & 49 & 89,1 \\
\hline Ruptura parcial & 6 & 10,9 \\
\hline Ruptura total & \multicolumn{2}{|l|}{} \\
\hline Local da lesão geral & 47 & 85,5 \\
\hline Supraespinhal & 6 & 10,9 \\
\hline Infraespinhal & 2 & 3,6 \\
\hline Subescapular &
\end{tabular}


Tabela 3 Comparação dos fatores demográficos, metabólicos e físicos em relação à ocorrência de lesão do manguito rotador

\begin{tabular}{|c|c|c|c|c|c|c|c|}
\hline & \multicolumn{3}{|c|}{$\begin{array}{l}\text { Lesão do manguito rotador } \\
(\mathrm{n}=55)\end{array}$} & \multicolumn{3}{|c|}{$\begin{array}{l}\text { Sem lesão do manguito rota- } \\
\text { dor }(n=180)\end{array}$} & \multirow[t]{2}{*}{ Valor de $p$} \\
\hline & $\mathrm{n}$ & Média & $\begin{array}{l}\text { ( } \pm \text { Desvio } \\
\text { padrão) }\end{array}$ & $\mathrm{n}$ & Média & $\begin{array}{l}\text { ( } \pm \text { Desvio } \\
\text { padrão) }\end{array}$ & \\
\hline \multicolumn{8}{|l|}{ Fatores demográficos } \\
\hline Gênero feminino & 36 & & & 121 & & & \\
\hline Gênero masculino & 19 & & & 59 & & & \\
\hline Idade (anos) & & 46 & 11,2 & & 38,6 & 10,4 & $<0,001$ \\
\hline \multicolumn{8}{|l|}{ Fatores metabólicos } \\
\hline $\mathrm{LDL}(\mathrm{mg} / \mathrm{dL})$ & & 100 & 35 & & 101 & 35 & 0,90 \\
\hline $\mathrm{HDL}(\mathrm{mg} / \mathrm{dL})$ & & 53,4 & 16 & & 48,2 & 10 & 0,026 \\
\hline Colesterol total (mg/dL) & & 186 & 40 & & 182 & 37 & 0,54 \\
\hline $\begin{array}{l}\text { Razão de hipertensão arterial sistêmica } \\
\text { (sim/não) }\end{array}$ & $33 / 55$ & & & $52 / 180$ & & & $<0,001$ \\
\hline Razão de diabeltes melito (sim/não) & $16 / 55$ & & & $26 / 180$ & & & 0,013 \\
\hline $\begin{array}{l}\text { Tempo de exposição } \\
\text { à obesidade (anos) }\end{array}$ & & 15 & 9,1 & & 10,7 & 7,5 & $<0,001$ \\
\hline \multicolumn{8}{|l|}{ Fatores físicos } \\
\hline Peso $(\mathrm{kg})$ & & 102 & 21 & & 102 & 22 & 0,94 \\
\hline Altura $(\mathrm{cm})$ & & 165 & 10 & & 167 & 11 & 0,38 \\
\hline Índice de massa corporal $\left(\mathrm{kg} / \mathrm{m}^{2}\right)$ & & 37,2 & 5,5 & & 36,6 & 5,7 & 0,54 \\
\hline Circunferência abdominal (cm) & & 114 & 15 & & 115 & 15 & 0,88 \\
\hline
\end{tabular}

Abreviaturas: LDL, low-density lipoprotein (lipoproteína de baixa densidade); HDL, high-density lipoprotein (lipoproteína de alta densidade). Nota: Os valores em negrito indicam diferença estatisticamente significativa.

o método de seleção das variáveis do tipo stepwise (passo a passo). Por fim, a curva característica de operação do receptor (COR) foi utilizada, a fim de identificar o melhor ponto de corte da variável idade para presença de lesão do MR. Os dados foram analisados utilizando o programa estatístico Statistical Package for the Social Sciences (SPSS, IBM Corp., Armonk, NY, EUA), versão 26. O nível de significância estatística foi estabelecido em $p<0,05$.

\section{Resultados}

As características demográficas, metabólicas e físicas, e o perfil das lesões do MR da amostra estão detalhados nas -Tabelas $\mathbf{1}$ e $\mathbf{2}$, respectivamente.

\section{Comparação de fatores demográficos, metabólicos e físicos em indivíduos obesos com e sem lesão do manguito rotador}

Os dados demonstraram que, no grupo com lesão, idade $(p<0,001)$, presença de DM $(p=0,013)$, HAS $(p<0,001)$, nível de $\operatorname{HDL}(p=0,026)$, e tempo de exposição à obesidade $(p<0,001)$ apresentaram resultados significativamente maiores em comparação ao grupo sem lesão (-Tabela $\mathbf{3}$ ).

Indivíduos com lesão do MR apresentaram em média 7,4 anos a mais de idade, uma prevalência maior de HAS e DM, de $31,3 \%$ e $15 \%$, respectivamente, além de cerca de 4,3 anos a mais de exposição à obesidade.

\section{Associação entre lesão de manguito rotador e obesidade}

Não foi evidenciada associação da lesão do MR com o IMC $(p=0,82)$, o tempo de exposição à obesidade $(p=0,29)$, ou a circunferência abdominal $(p=0,52)$ na amostra estudada, avaliada por regressão logística binária (-Tabela 4). Além disso, não existiu correlação significativa entre o IMC (rs = 0,$029 ; p=0,83$ ) e o tempo de exposição à obesidade (rs $=0,061 ; p=0,66)$ e a gravidade da lesão do MR.

Tabela 4 Parâmetros associados para lesão do manguito rotador avaliados por regressão logística binária

\begin{tabular}{|l|l|l|l|l|}
\hline & Coeficiente & $\begin{array}{l}\text { Razão } \\
\text { de } \\
\text { chances }\end{array}$ & $\begin{array}{l}\text { Intervalo } \\
\text { de } \\
\text { confiança } \\
\text { de 95\% }\end{array}$ & $\begin{array}{l}\text { Valor } \\
\text { de } p\end{array}$ \\
\hline \multicolumn{3}{|l|}{ Fatores demográficos } & $1,03-1,09$ & $\mathbf{0 , 0 0 0 3}$ \\
\hline $\begin{array}{l}\text { Idade } \\
\text { (anos) }\end{array}$ & 0,057 & 1,06 & & \\
\hline Fatores metabólicos & 2,68 & $1,38-5,20$ & $\mathbf{0 , 0 0 4}$ \\
\hline $\begin{array}{l}\text { Hipertensão } \\
\text { arterial } \\
\text { sistêmica }\end{array}$ & 0,984 & & & \\
\hline
\end{tabular}

Notas: Os valores em negrito: indicam diferença estatisticamente significativa. O método de seleção de variáveis foi avançar passo a passo, no nível 5\%. 


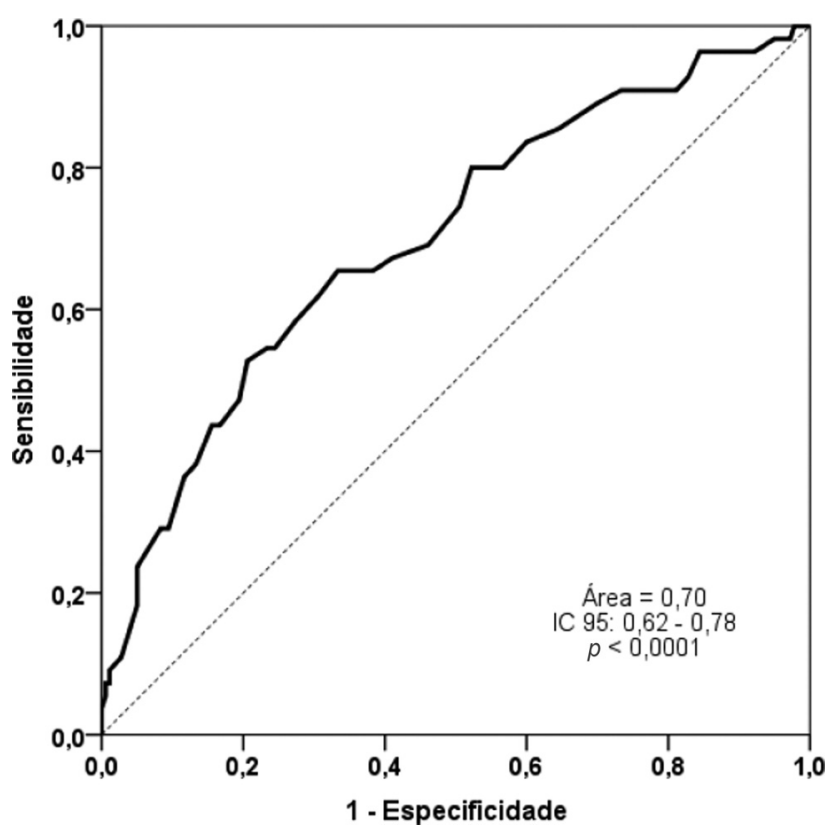

Fig. 2 Curva característica de operação do receptor (COR). Identificação do ponto de corte da variável idade para presença de lesão do manguito rotador $(\mathrm{MR}): \geq 43$ anos.

\section{Associação entre fatores demográficos e metabólicos com a lesão do manguito rotador}

Na busca por demais parâmetros associados (demográficos e metabólicos) de forma independente à lesão do MR, foi observada associação com idade $(p=0,0003)$ e HAS ( $p$ $=0,004)$ ( - Tabela 4), o que demonstra que, quanto maior a idade e a presença de HAS, maior a probabilidade de lesão do MR. Utilizando a metodologia COR, com sensibilidade de $65,5 \%$ e especificidade de $66,7 \%$, determinamos que uma idade $\geq 43$ anosera um ponto de corte para presença de lesão do MR (-Figura 2).

\section{Discussão}

A relação da obesidade com lesões do sistema musculoesquelético, como as tendinopatias, são o foco de estudos e controvérsias científicas. ${ }^{9-11,13,21}$ Pesquisas recentes ${ }^{11}$ sugerem uma associação importante entre lesões tendíneas e demais distúrbios metabólicos (HAS e DM).

Diante disso, analisamos a relação entre a presença e a gravidade da lesão do MR e a obesidade e o tempo de exposição à obesidade. De forma secundária, avaliamos a relação e a prevalência de fatores demográficos e demais fatores metabólicos em indivíduos obesos com lesão do MR. Neste estudo epidemiológico transversal, não foi demonstrada associação entre a ocorrência e a gravidade da lesão do MR e o IMC, a circunferência abdominal, ou o tempo de exposição à obesidade. No entanto, indivíduos com lesão demonstraram uma exposição a anos de obesidade e prevalência de doenças metabólicas (HAS e DM) maiores do que pacientes sem lesão. Por fim, a HAS e a idade avançada foram fatores relacionados com a presença de lesão do MR.

A maior suscetibilidade a rupturas e degenerações tendíneas em indivíduos com valores altos de IMC e circunferência abdominaltem uma explicação fisiológica, ligada ao aumento da produção de adipocinas (fator de necrose tumoral alfa, leptina, adiponectina, angiotensinogênio, e interleucinas 6, 8 , 10 e 18), que leva a estresse oxidativo, inflamação, disfunção endotelial e apoptose celular. ${ }^{9,11,14} \mathrm{O}$ primeiro estudo de caso controle a estabelecer tal associação clínica entre a ocorrência e gravidade de lesões do MR com a obesidade foi conduzido por Gumina et al. ${ }^{9}$ Os autores avaliaram 381 pacientes, e demonstraram que indivíduos com IMC médio de $30 \mathrm{~kg} / \mathrm{m}^{2}$ apresentavam mais do que o dobro de lesões do que indivíduos não obesos. Além disso, quanto maior o IMC, maior o grau das lesões do MR evidenciadas. No entanto, Titchener et al.,22 em pesquisa com volumosa amostra (5 mil pacientes), concluíram que somente o sobrepeso (IMC de $25,1 \mathrm{~kg} / \mathrm{m}^{2}$ a $30 \mathrm{~kg} / \mathrm{m}^{2}$ ) estava significativamente associado à lesão do MR. Os pacientes com IMC $>30 \mathrm{~kg} / \mathrm{m}^{2}$ não demonstraram correlação estatística. No presente estudo, centrado apenas em indivíduos com IMC $>30$ $\mathrm{kg} / \mathrm{m}^{2}$, constatamos que a obesidade não se apresentou como fator associado à prevalência ou à gravidade da lesão do MR. Porém, o tempo de exposição à obesidade foi maior nos pacientes com lesão. Além disso, evidenciamos uma antecipação na média ( 46 anos) e no ponto de corte (43 anos) da idade para o aparecimento de rupturas dos tendões de 12 à 15 anos, quando comparada a estudos anteriores. ${ }^{29}$ Yamaguchi et al. ${ }^{29}$ demonstram que a idade média para a ocorrência de algum nível de ruptura é de 58 anos, e de 68 anos para ruptura total. $O$ fato de nossa amostra conter apenas indivíduos obesos pode ser uma possível explicação para a diferença observada e sugerir que a obesidade antecipe o aparecimento das rupturas tendíneas.

De forma semelhante, os níveis elevados de açúcar no sangue têm influência sobre a saúde dos tendões. ${ }^{17}$ Rupturas graves são seis vezes mais comuns na presença de mais de um fator de risco cardiovascular, como DM e HAS. ${ }^{20}$ Nossos resultados demonstram uma prevalência maior de disfunções metabólicas (DM, HAS) em pacientes com lesão do MR, e uma importante associação entre HAS e a lesão tendínea. As diminuições da angiogênese, da proliferação de fibroblastos, da síntese de colágeno, e da liberação de fatores de crescimento são resultados deletérios observados em tendões de diabéticos, que reduzem a capacidade biomecânica tecidual e aumentam a predisposição ao desenvolvimento ou agravamento de tendinopatias. ${ }^{17,18}$ Em estudo envolvendo análise do MR por ultrassonografia em pacientes com diabetes, Abate et al. ${ }^{30}$ demonstraram que até mesmo os indivíduos assintomáticos apresentam padrões de degeneração mais avançada, principalmente no tendão supraespinhal, do que indivíduos sem diagnóstico de diabetes. Gumina et al., ${ }^{19}$ em pesquisa envolvendo 400 pacientes, concluíram que indivíduos hipertensos tinham de 2 a 4 vezes mais chances de sofrer rupturas grandes (envolvendo um tendão inteiro) e massivas (mais de 2 tendões) do MR em comparação com indivíduos normotensos. As principais explicações para essa importante evidência recaem sobre o estado de hipóxia tendínea gerada pelo mecanismo hipertensivo, ${ }^{20}$ mesmo em indivíduos em tratamento farmacológico, uma vez que grande parte dos fármacos hipertensivos parece ter maior ação nos grandes vasos, mantendo a hipóxia no tecido tendineo e favorecendo, assim, a degeneração tecidual. ${ }^{19}$ 
Os achados do presente estudo devem ser tratados com cautela devido às limitações metodológicas. São necessários estudos de caso-controle e coorte bem controlados em larga escala para obter uma estimativa precisa da prevalência/incidência e das associações de fatores demográficos, metabólicos e físicos com o risco de desenvolvimento de lesão do MR. Além disso, esses resultados devem ser extrapolados para a população em geral com cuidado, uma vez que a amostra do estudo se limitou aos pacientes de um ambulatório específico. Defendemos a necessidade de futuros trabalhos maximizarem a análise não somente da obesidade, como também do efeito crônico desta comorbidade (tempo de exposição), como fator determinante na geração de disfunções musculoesqueléticas.

\section{Conclusão}

Não evidenciamos associação entre a obesidade e o tempo de exposição à obesidade e a ocorrência e a gravidade da lesão do MR. Porém, os indivíduos com lesão apresentaram maior tempo de exposição à obesidade e prevalência de disfunções metabólicas (DM e HAS) do que indivíduos sem lesão do MR. Além disso, nossos achados sugerem uma importante associação entre HAS e idade avançada e lesão do MR nesta população de indivíduos obesos.

\section{Suporte Financeiro}

Não houve suporte financeiro de fontes públicas, comerciais, ou sem fins lucrativos.

\section{Conflito de Interesses}

Os autores declaram não haver conflito de interesses.

\section{Referências}

1 Aronow WS. Association of obesity with hypertension. Ann Transl Med 2017;5(17):350

2 Al-Goblan AS, Al-Alfi MA, Khan MZ. Mechanism linking diabetes mellitus and obesity. Diabetes Metab Syndr Obes 2014; 7:587-591

3 Loures FB, de Araújo Góes RF, Labronici PJ, Barretto JM, Olej B. Evaluation of body mass index as a prognostic factor in osteoarthrosis of the knee. Rev Bras Ortop 2016;51(04):400-404

4 Afshin A, Forouzanfar MH, Reitsma MB, et al. Health Effects of Overweight and Obesity in 195 Countries over 25 Years. N Engl J Med 2017;377(01):13-27

5 Brasil. Ministério da Saúde. Vigitel Brazil 2018: surveillance of risk and protective factors for chronic diseases by telephone survey: estimates of frequency and sociodemographic distribution of risk and protective factors for chronic diseases in the capitals of the 26 Brazilian states and the Federal District in 2018. Brasilia: Editora MS; 2018

6 Melin EO, Thulesius HO, Hillman M, Landin-Olsson M, Thunander M. Abdominal obesity in type 1 diabetes associated with gender, cardiovascular risk factors and complications, and difficulties achieving treatment targets: a cross sectional study at a secondary care diabetes clinic. BMC Obes 2018;15(05)

7 Patel SA, Ali MK, Alam D, et al. Obesity and its Relation With Diabetes and Hypertension: A Cross-Sectional Study Across 4 Geographical Regions. Glob Heart 2016;11(01):71-79.e4

8 Engin A. The Definition and Prevalence of Obesity and Metabolic Syndrome. Adv Exp Med Biol 2017;960:1-17
9 Gumina S, Candela V, Passaretti D, et al. The association between body fat and rotator cuff tear: the influence on rotator cuff tear sizes. J Shoulder Elbow Surg 2014;23(11):1669-1674

10 Walsh TP, Arnold JB, Evans AM, Yaxley A, Damarell RA, Shanahan EM. The association between body fat and musculoskeletal pain: a systematic review and meta-analysis. BMC Musculoskelet Disord 2018;233(19)

11 Franceschi F, Papalia R, Paciotti M, et al. Obesity as a risk factor for tendinopathy: a systematic review. Int J Endocrinol 2014; 2014:1-10

12 Corbin LJ, Richmond RC, Wade $\mathrm{KH}$, et al. Body mass index as a modifiable risk factor for type 2 diabetes: Refining and understanding causal estimates using Mendelian randomisation. Diabetes 2016;65(10):3002-3007

13 Özkuk K, Ateş Z The effect of obesity on pain and disability in chronic shoulder pain patients. J Back Musculoskeletal Rehabil 2020;33(01):73-79

14 Scott A, Zwerver J, Grewal N, et al. Lipids, adiposity and tendinopathy: is there a mechanistic link? Critical review. Br J Sports Med 2015;49(15):984-988

15 Burne G, Mansfield M, Gaida JE, Lewis JS. Is there an association between metabolic syndrome and rotator cuff-related shoulder pain? A systematic review. BMJ Open Sport Exerc Med 2019;5 (01):e000544

16 Sayampanathan AA, Andrew THC. Systematic review on risk factors of rotator cuff tears. J Orthop Surg (Hong Kong) 2017;25 (01)

17 Ahmed AS. Does Diabetes Mellitus Affect Tendon Healing? Adv Exp Med Biol 2016;920:179-184

18 Lui PPY. Tendinopathy in diabetes mellitus patients-Epidemiology, pathogenesis, and management. Scand J Med Sci Sports 2017;27(08):776-787

19 Gumina S, Arceri V, Carbone S, et al. The association between arterial hypertension and rotator cuff tear: the influence on rotator cuff tear sizes. J Shoulder Elbow Surg 2013;22(02): 229-232

20 Djerbi I, Chammas M, Mirous MP, Lazerges C, Coulet BFrench Society For Shoulder and Elbow (SOFEC) Impact of cardiovascular risk factor on the prevalence and severity of symptomatic fullthickness rotator cuff tears. Orthop Traumatol Surg Res 2015;101 (6Suppl)S269-S273

21 Lewis J. Rotator cuff related shoulder pain: Assessment, management and uncertainties. Man Ther 2016;23:57-68

22 Titchener AG, White JJ, Hinchliffe SR, Tambe AA, Hubbard RB, Clark DI. Comorbidities in rotator cuff disease: a case-control study. J Shoulder Elbow Surg 2014;23(09):1282-1288

23 Cochran WG. Sampling Techniques. 3rd ed. New Jersey: Wiley; 1977

24 Jobe FW, Jobe CM. Painful athletic injuries of the shoulder. Clin Orthop Relat Res 1983;(173):117-124

25 Holtby R, Razmjou H. Validity of the supraspinatus test as a single clinical test in diagnosing patients with rotator cuff pathology. J Orthop Sports Phys Ther 2004;34(04):194-200

26 Jain NB, Wilcox RB III, Katz JN, Higgins LD. Clinical examination of the rotator cuff. PM R 2013;5(01):45-56

27 Selvaraj SS, Kiran Das S, Murthy N, Patil V, Hiremath R. Diagnostic accuracy of ultrasound vs. MRI in evaluation of rotator cuff injuries. Int J Radiol Diag Imag 2019;2(02):92-95

28 El-Kouba G, Andreas Huber T, Freitas JR, Steglich V, Ayzemberg H, Santos AM. Comparison of complementary exams in the diagnosis of rotator cuff injuries. Rev Bras Ortop 2015;45(05):418-425

29 Yamaguchi K, Ditsios K, Middleton WD, Hildebolt CF, Galatz LM, Teefey SA. The demographic and morphological features of rotator cuff disease. A comparison of asymptomatic and symptomatic shoulders. J Bone Joint Surg Am 2006;88(08):1699-1704

30 Abate M, Schiavone C, Salini V. Sonographic evaluation of the shoulder in asymptomatic elderly subjects with diabetes. BMC Musculoskelet Disord 2020;278(11) 